\title{
Verteilungswirkungen von Staatsschulden
}

\author{
Carl Christian von Weizsäcker
}

Online publiziert: 8. Oktober 2018

(C) Der/die Autor(en) 2018

Zusammenfassung Es wird eine Methode vorgeschlagen, mit deren Hilfe in den betrachteten hypothetischen Fällen eindeutige Verteilungsergebnisse von Staatsschulden abgeleitet werden können. Ausgangspunkt ist eine verallgemeinerte „Golden Rule of Accumulation“. Nimmt man den Golden Rule Pfad als „Benchmark“ kann man für Abweichungen von ihm eindeutige Verteilungsergebnisse von Staatsschulden feststellen. Das geht sowohl für die Verteilung zwischen verschiedenen Generationen als auch für die Wohlstandsverteilung innerhalb einer Generation.

Schlüsselwörter Staatsschulden · Intergenerationelle Verteilung · Intragenerationelle Verteilung

JEL Code H63 $\cdot$ D31 $\cdot$ E14

\section{Distribution effects of public debt}

Abstract I propose a method by which one can derive unambiguous distribution effects of public debt policies. The benchmark is a generalized "Golden Rule of Accumulation" path. Certain deviations from that path by changes in public debt policies have unambiguous distributional effects. I provide examples for this method for the income distribution between generations as well as for the income distribution within any given generation.

Keywords Public debt $\cdot$ Distribution between generations $\cdot$ Distribution within a generation

\footnotetext{
Vortrag Wirtschaftspolitischer Ausschuss 2018 in Witten

C. C. von Weizsäcker $(\bowtie)$

Max Planck Institut zur Erforschung von Gemeinschaftsgütern, Bonn, Deutschland

E-Mail: weizsaecker@coll.mpg.de
} 


\section{Einleitung}

Die Verteilungswirkungen von Staatsschulden festzustellen, ist nicht einfach. Daher beschränke ich mich darauf, eine bestimmte Methode vorzuschlagen, mit deren Hilfe man ohne zu großen empirisch-ökonometrischen Aufwand in einfachen Fällen recht robuste Ergebnisse erzielen kann. Der Vorteil dieser Methode ist die Eindeutigkeit der Ergebnisse. Der offensichtliche Nachteil ist, dass man die Ergebnisse nicht unmittelbar auf die große Komplexität der Wirklichkeit anwenden kann.

Ich unterscheide zwischen Intergenerationseffekten von Staatsschulden einerseits und Intragenerationseffekten andererseits. Im folgenden Abschn. 2 behandle ich die Intergenerationseffekte. In den Abschn. 3 und 4 behandle ich zwei Fälle von Intragenerationseffekten.

Diese beiden theoretischen Fälle habe ich so gewählt, dass sie zu gegenteiligen Ergebnissen führen. Das soll zeigen, dass es eine einfache Pauschalantwort auf die Frage nach den Verteilungswirkungen von Staatsschulden nicht gibt.

In dem verfügbaren Raum kann ich nicht auf solche Effekte der Staatsschulden eingehen, die sich im Zusammenhang mit der Konjunkturpolitik ergeben, sprich: auf die Funktion der Staatsschulden als antizyklisch wirkender Stabilisator oder gar bewusster antizyklischer Konjunkturpolitik. Auch durchaus problematische Übertreibungen der staatlichen Schuldenpolitik können hier nicht thematisiert werden.

\section{Verteilungswirkungen von Staatsschulden auf unterschiedliche Generationen}

Ich betrachte primär Steady-State-Zustände. Diese zeichnen sich dadurch aus, dass die Schuldenquote im Zeitverlauf konstant bleibt. Es werden dann unterschiedliche Steady State Zustände verglichen, die sich - ceteris paribus -durch unterschiedlich hohe Staatsschuldenquoten unterscheiden. Der methodische Grundgedanke ist das Allgemeine Gleichgewicht. Ich arbeite mit dem Modellgedanken der sich überlappenden Generationen. Zur Vereinfachung behandle ich ein Modell einer geschlossenen Volkswirtschaft.

Vorbild für ein derartiges Herangehen an die Fragestellung ist der Artikel von Diamond (1965), der bekanntlich zu den meistgelesenen Arbeiten des Faches zählt. Allerdings ist man heute nicht darauf angewiesen, die Analyse, wie bei Diamond, mit dem Solow-Wachstumsmodell durchzuführen. Mit Hilfe der Kapitaltheorie ist es möglich, die Aussagen sehr viel breiter abzustützen. Indessen verzichte ich hier darauf, das mathematische Modell in seiner Gänze darzustellen.

Ich skizziere hier eine Verallgemeinerung der Goldenen Regel der Akkumulation: Ich brauche nicht vorauszusetzen, dass das reale Wachstum der Volkswirtschaft in eindeutiger Weise gemessen werden kann. Wir kennen ja das Indexproblem, das es uns verunmöglicht, eine säkular gültige durchschnittliche Wachstumsrate des realen Sozialprodukts eindeutig zu messen. Vgl. hierzu Weizsäcker (2015). Ich betrachte eine geschlossene Volkswirtschaft mit einer vorgegebenen konstant bleibenden Wachstumsrate des nominellen Sozialprodukts bzw. Volkseinkommens. Der Komplexitätsgrad des Produktionssystems ist beliebig, solange damit ein Allgemeines 
Gleichgewicht möglich ist. Auf den einzelnen Märkten braucht nicht vollständige Konkurrenz zu herrschen. Ein Alternativmodell könnte auf mikroökonomischer Ebene das der monopolistischen Konkurrenz sein. Durch geeignete Wahl der Staatsschuldenquote hat es die Politik in der Hand, wie hoch der gleichgewichtige Zinssatz ist, der im Zeitverlauf konstant bleibt.

Ich unterstelle, dass der risikofreie nominelle Kapitalmarktzins sowohl auf der Kapitalnachfrageseite (Investitionen der Unternehmen und Haushalte) als auch auf der Kapitalangebotsseite (Spartätigkeit der privaten Haushalte und der Unternehmen) im Steady State Vergleich ein korrektes Preissignal darstellt.

Damit meine ich folgendes: Auf der Kapitalnachfrageseite: die nominelle Wachstumsrate der Volkswirtschaft $g$ ist vorgegeben und ist in allen betrachteten Steady States dieselbe. Sie wird durch die Liquiditätsversorgung der Volkswirtschaft seitens der Zentralbank gesteuert. Bei jedem gegebenem, zeitlich konstant bleibendem Zinssatz $r$ verwirklicht sich ein Produktionssystem $\wp(r)$. Es sei $y$ das nominelle Sozialprodukt, das für alle Steady States in einem gegebenen Zeitpunkt den gleichen Wert annimmt. Es sei $v(r)$ das im Produktionsprozess gebundene Kapital in nominellen Geldeinheiten gemessen. Es sei $w(r)=y-r v(r)$ das Sozialprodukt abzüglich der Zinskosten des eingesetzten Kapitals. $w(r)$ ist der Überschuss der mittels des Kapitaleinsatzes erwirtschafteten Sozialprodukts über den Kosten dieses Kapitaleinsatzes. Definition: Im Steady State Vergleich ist $r$ ein korrektes Preissignal, wenn gilt: Für jedes $\bar{r}$ gilt die Ungleichung

$$
w(\bar{r}, \wp(\bar{r})) \geq w(\bar{r} . \wp(r)) \text { für alle } r
$$

Mit anderen Worten: wir sprechen auf der Kapitalnachfrageseite davon, dass der risikofreie Zinssatz im Steady State Vergleich ein korrektes Preissignal ist, wenn der Wettbewerb zwischen den Produktionssystemen dazu führt, dass dasjenige Produktionssystem gewählt wird, das den Überschuss der Wertschöpfung jenseits der Zinskosten maximiert. In den Vergleich der Produktionssysteme wird aber nur die Menge der Produktionssysteme einbezogen, die bei einem Steady State Zinssatz zum Zuge kommen. Ich brauche somit nicht vorauszusetzen, dass das bei dem herrschenden Steady State Zinssatz gewählte Produktionssystem auch solche praktikable Produktionssysteme schlägt, die bei anderen institutionellen Voraussetzungen gewählt werden würden. Das bei einem bestimmten Steady State Zinssatz gewählte Produktionssystem muss also nicht Pareto-optimal sein. Entscheidend ist nur, dass es den Sieg gegenüber einer bestimmten Teilmenge von praktikablen Produktionssystemen davon trägt.

Auf der Kapitalangebotsseite arbeite ich mit der Konstruktion eines repräsentativen Haushalts, der sich gemäß dem Modell der überlappenden Generationen für jede Alterskohorte quasi wiederholt. Für jeden zeitlich gleichbleibenden Zinssatz r setzt sich bei der im Gleichgewicht dazugehörigen Staatsschuldenquote $D(r)$ ein Einkommen der privaten Haushalte ohne Zinseinkünfte $\widehat{w}(r)$ durch, das durch folgende Gleichung charakterisiert ist:

$$
\widehat{w}(r)=w(r)-(r-g) D
$$


Hierbei sind einbehaltene Gewinne der Unternehmen ihren Anteilseignern prorata zugerechnet. Man kann sich diese Gleichung am besten klarmachen, indem man annimmt, dass der Steady-State-Primärüberschuss des Fiskus abzüglich die SteadyState-Nettoneuverschuldung des Fiskus, also $(r-g) D$, durch eine vom repräsentativen Haushalt zu entrichtende Steuer entsteht. Dieser Ausdruck kann natürlich auch negativ sein, dann nämlich, wenn entweder $r<g$ und $D>0$ oder $r>g$ und $D<0$ gilt.

Es sei $\eta(r)$ das Arbeit-Konsumpattern des repräsentativen Haushalts, das beim Gleichgewichtszinssatz $r$ verwirklicht wird. Es sei $U(\eta)$ der Lebensnutzen, der aus dem Arbeit-Konsumpattern $\eta$ resultiert. Es sei $\widetilde{w}(r ; \eta)$ das Einkommen (ohne Zinseinkommen), das erforderlich ist, um das Pattern $\eta$ beim Zinssatz $r$ zu finanzieren. Es sei $\operatorname{Eta}(\bar{U})$ die Menge der Patterns $\eta$, deren Lebensnutzen $U(\eta)$ strikt höher ist als $\bar{U}$.

Definition Der Zinssatz ist ein korrektes Preissignal auf der Kapitalangebotsseite, wenn folgende Ungleichung gilt:

$$
\widehat{w}(r)<\widetilde{w}(r ; \eta) \text { für alle } \eta \in \operatorname{Eta}(U(\eta(r)))
$$

Die ökonomische Bedeutung dieser Ungleichung und damit der Annahme eines korrekten Preissignals ist, dass der repräsentative Haushalt für das ihm vorgegebene intertemporale Budget seinen Lebensnutzen maximiert. Er kann daher kein Pattern $\eta$ erreichen, das einen höheren Lebensnutzen als den erreichten stiftet.

Nun kann man zeigen: im Vergleich der verschiedenen Steady States wird der Lebensnutzen des repräsentativen Haushalts bei dem nominalen Zinssatz maximiert, der gleich der vorgegebenen nominalen Wachstumsrate des Systems ist. Das ist die stark verallgemeinerte „Goldene Regel der Akkumulation“ (vgl. Phelps 1961; von Weizsäcker 1962).

Die Beweisidee ist sehr einfach: $\mathrm{Zu}$ diesem Zweck führen wir die Größe $\dddot{w}(g ; \wp(r))$ ein: sie stellt das hypothetische Einkommen ohne Zinseinkommen des repräsentativen Haushalts dar, das sich ergeben würde, wenn der tatsächliche Zinssatz gleich $g$ ist, zugleich aber das Produktionssystem verwirklicht ist, das bei einem Zins $r$ verwirklicht würde. Da der tatsächliche Zinssatz gleich $g$ ist, fällt der Unterschied zwischen $\dddot{w}(g ; \wp(r))$ und dem von den Unternehmen erwirtschafteten Überschuss $w(g ; \wp(r))$ weg. Bei jedem Produktionssystem $\wp(r)$ gilt gemäß der Annahme eines korrekten Preissignals $r$ auf der Kapitalnachfrageseite, dass

$$
w(g ; \wp(r)) \leq w(g ; \wp(g))
$$

Daher gilt auch

$$
\dddot{w}(g ; \wp(r)) \leq w(g ; \wp(g))
$$


Aus dem angenommenen korrekten Preissignal auf der Kapitalangebotsseite ergibt sich damit die Ungleichung

$$
\dddot{w}(g ; \wp(r)) \leq w(g ; \wp(g))=\widehat{w}(g)<\widetilde{w}(r ; \eta) \text { für alle } \eta \in \operatorname{Eta}(U(\eta(g)))
$$

Sie zeigt, dass der Lebensnutzen beim Produktionssystem $\wp(r)$ nicht größer sein kann als beim Produktionssystem $\wp(g)$. QED.

Das wohlfahrtsökonomische Potential dieser Verallgemeinerung der Goldenen Regel der Akkumulation ist bisher nicht erkannt worden. Ich vermute, dass es sehr groß ist und auch für aktuelle makro-ökonomische Debatten relevant ist. Ich erwähne als ein Beispiel nur, dass Steady State Aussagen mit nominalen Größen möglicherweise kompatibel mit dem Strukturwandel bei den realen, disaggregierten Größen gemacht werden können.

Ein weiteres Ergebnis dieses Ansatzes ist folgendes: Ist der Zinssatz im Steady State gleich der Wachstumsrate des Systems (beides als nominelle Größen), dann gilt im einfachsten Fall dort die Gleichung

$$
Z=D+T
$$

wobei $Z$ die durchschnittliche Warteperiode des repräsentativen Haushalts, $T$ die durchschnittliche Produktionsperiode (à la Böhm-Bawerk 1889 - aber gemäß Hicks 1939 modernisiert) und $D$ die auf den gesamtwirtschaftlichen Konsum bezogene Staatsschuldenquote ist. Die Warteperiode ist der durchschnittliche zeitliche Abstand zwischen dem Konsum und dem Arbeitseinkommen des repräsentativen Haushalts. Ihr entspricht in jeder Periode der durchschnittliche Vermögensbestand der sich überlappenden Generationen. Der durchschnittlichen Produktionsperiode entspricht die durchschnittliche Kapitalbindung der Unternehmen. Das private Vermögen verteilt sich somit auf den Kapitalbestand des Produktionssektors und auf die Staatsschulden.

Im komplizierteren Fall führt man zusätzlich kapitalisierte Bodenrenten und Monopol- bzw. Oligopolrenten als private Vermögensbestandteile ein. Dann ergibt sich die folgende Formel

$$
Z=D+T+\beta l
$$

Dabei ist $\beta$ der Anteil der genannten Renten am gesamtwirtschaftlichen Konsum und $l$ die ,price earnings ratio“ dieser Renten. Sie ist quasi die risikogewichtete, subjektiv vom Kapitalmarkt wahrgenommene „Lebenserwartung“ des privaten Eigentums an diesen Renten. Die Größe $\beta l$ ist damit die kapitalisierte Rente $\beta$. Sowohl der Homburgsche Bodenrenten-Effekt (Homburg 1991), (modifiziert durch das Wertänderungsrisiko und das Enteignungsrisiko) als auch Abweichungen des Tobin-Q vom Wert 1 sind damit in dem Term $\beta l$ enthalten. Auch diese Formel gilt nur dann, wenn der Zinssatz gleich der Wachstumsrate ist. Man kann zeigen: Auch dann gilt, dass ein Zinssatz gleich der Wachstumsrate den Lebensnutzen des repräsentativen Haushalts maximiert. 
Die hier vorgeführte Analyse anhand des repräsentativen Haushalts reicht natürlich nicht für eine Theorie der Verteilungseffekte von Staatsschulden. Indessen eignet sie sich schon einmal, um die Problematik der Aussage aufzuzeigen: „Staatsschulden verschieben die Lasten von der gegenwärtigen Generation auf zukünftige Generationen“. Im Rahmen einer Steady State-Analyse ist diese Aussage falsch. Denn hier bedeutet jede Verbesserung für die gegenwärtige Generation zugleich eine Verbesserung für alle künftigen Generationen. In der Steady State Analyse hat eine heutige Nettoneuverschuldung ein Abbild in jedem künftigen Jahr.

Natürlich kann man von dieser Steady State Analyse abweichen. Man kann die Frage stellen: wie wirkt sich eine heutige zusätzliche Nettoneuverschuldung jenseits des Konstant-Haltens der Schuldenquote $D$ auf künftige Generationen aus? Sofern eine intertemporale Budgetbeschränkung für den Staat gilt, muss ein heutiges Schuldenmachen des Staats zu einer zusätzlichen Belastung künftiger Generationen führen. Das ist der Fall der sogenannten „Ricardianischen Äquivalenz“. Sie gilt dann, wenn der Gegenwartswert der Summe aller Nettoneuverschuldung (positiv oder negativ) endlich ist. Ist er das, dann muss er Null sein, woraus sich ableiten lässt, dass mehr Schuldenmachen heute durch mehr Schuldenrückzahlung in der Zukunft kompensiert werden muss.

Diese Ricardianische Äquivalenz gilt, wenn der Zinssatz nachhaltig höher ist als die Wachstumsrate des Systems. Die Ricardianische Äquivalenz gilt nicht, wenn der Zinssatz nachhaltig unter der Wachstumsrate des Systems liegt: der Fall der „dynamischen Ineffizienz“. Der Golden Rule Pfad mit der Gleichheit von Zins und Wachstumsrate ist die Grenze für den Gültigkeitsbereich der Ricardianischen Äquivalenz.

Solange der Zinssatz ein korrektes Preissignal ist, kommen wir damit zu dem Zwischenergebnis: ist der gleichgewichtige Zinssatz höher als die Wachstumsrate, geht eine erhöhte heutige Nettoneuverschuldung zulasten künftiger Generationen. Dies ist nicht der Fall, wenn „dynamische Ineffizienz“ vorliegt, das heißt, wenn der Zinssatz niedriger ist als die Wachstumsrate. Ist der Zinssatz gleich der Wachstumsrate, dann spricht einiges dafür, die Nettoneuverschuldung so zu wählen, dass die Schuldenquote konstant bleibt.

Eine vertiefte normative Wertung sollte im Rahmen einer Steady-State-Analyse auch die Perspektiven des realen Wirtschaftswachstums mit einbeziehen. In diesem Fall ist es auch nicht mehr so klar, ob bei einem Zins oberhalb der Wachstumsrate eine Reduktion der heutigen Nettoneuverschuldung angesagt ist. Auch wenn diese zulasten künftiger Generationen geht, mag es im Rahmen einer gesellschaftlichen Präferenz für mehr Gleichheit sinnvoll sein, den Lebensstandard der heutigen Generation zu steigern - sofern man davon ausgehen kann, dass es künftigen Generationen besser geht als der gegenwärtigen. Für diese Frage ist es dann wichtig, dass wir das reale wirtschaftliche Wachstum zumindest approximativ korrekt messen.

Zur Veranschaulichung dieser letzten Überlegung sei auf den technischen Fortschritt verwiesen, nicht nur jenen, der heute unter dem Stichwort „Digitalisierung“ zusammengefasst wird. Aber gerade auch die Digitalisierung bewirkt eine Änderung des Konsum-Warenkorbs in der Weise, dass sie die „Jungen“ gegenüber den „Alten“ privilegiert. Wenn zum Beispiel durch das Internet der Einzelhandel sich in Richtung „Amazon“ etc. verschiebt und dadurch manch stationäres Fachhandelsgeschäft 
schließt, so ist das für die „Jungen“ ein Vorteil, dem aber bei manchen „Alten“ der Nachteil gegenüber steht, dass sie nicht mehr so gut in der Form einkaufen können, die sie gewohnt sind. Analoge Effekte hat es ein bis zwei Generationen früher durch die Motorisierung gegeben. Indem die durch den PKW bewirkte lokale Mobilität sich erhöht hat, ist im stationären Einzelhandel die Wettbewerbsintensität gestiegen, sind entsprechend die Margen gesunken. Das aber begünstigt die Autofahrer, also damals primär die junge Generation, während die alten „Nicht-Autofahrer“ (oft ohne Führerschein) Einkaufsmöglichkeiten einbüßten, weil viele „Tante-EmmaLäden“ zumachten.

Ganz generell begünstigt der technische Fortschritt die jeweils junge Generation stärker als die jeweils ältere Generation. Wenn dieser Verteilungseffekt dadurch kompensiert wird, dass der Staat sich zugunsten der heutigen Bürger und zulasten künftiger Bürger verschuldet, so kann dies auch als ,,verteilungs-gerecht“ empfunden werden, da es den künftigen Bürgern ohnehin besser gehen wird als den gegenwärtigen.

Aber ich betone noch einmal, dass unter Bedingungen dynamischer Ineffizienz eine heutige zusätzliche Staatsverschuldung ohnehin nicht durch ein Opfer künftiger Generationen kompensieret werden muss. Eine Erhöhung der Staatsverschuldung und damit des Zinssatzes - mit dem Ziel dem Golden-Rule-Pfad näher zu kommen - ist damit unproblematisch, soweit es die inter-generationelle Verteilung betrifft.

\section{Intra-generationelle Verteilungseffekte - Fall 1}

Ich mache nunmehr zwei Gedankenexperimente, um festzustellen, wie sich unterschiedlich hohe Staatsschulden auf zwei Gruppen oder Klassen auswirken, die derselben Generation angehören. Im ersten Gedankenexperiment unterstelle ich das einfache Modell, für das im Fall $r=g$ die schon genannte Gleichung

$$
Z=T+D
$$

gilt. Wie schon mitgeteilt gilt die verallgemeinerte Goldene Regel der Akkumulation: der Lebensnutzen des „repräsentativen Haushalts“ ist hier höher als er es bei einem Steady State mit $r \neq g$ wäre.

Nun müssen wir uns noch in Erinnerung rufen, dass der Steady-State-Zins umso höher ist, je höher die Staatsschulden $D$ sind. Damit wissen wir, dass die partielle Ableitung des Lebenseinkommens des repräsentativen Haushalts nach dem SteadyState-Zins das gleiche Vorzeichen hat wie partielle Ableitung des Lebenseinkommens (d.h. des Lebensnutzens) des repräsentativen Haushalts nach den Steady State Staatsschulden. An der Stelle $r=g$ ist diese Ableitung somit Null. Eine ,infinitesimal kleine“ Erhöhung oder Senkung der Steady-State-Staatsschulden hat damit keinen Effekt auf das Lebenseinkommen des repräsentativen Haushalts.

Nun spalten wir den repräsentativen Haushalt auf in zwei Klassen: die „Reichen“ und die „Armen“. Die „Reichen“ verfügen über ein höheres Vermögen als die Armen. Eine ,,infinitesimal kleine“ Erhöhung der Steady-State-Staatsschulden und damit des Steady-State-Zinssatzes über $g$ hinaus bedeutet damit einen Gewinn der 
Reichen und einen gleich hohen Verlust der Armen. Denn in der Summe sind die Lebenseinkommen der beiden Klassen ja gleich geblieben.

Ist man in diesem einfachen Fall daran interessiert, die „Armen“ durch Staatsschuldenpolitik besser zu stellen (und sei es auf Kosten der „Reichen“), dann ist es angebracht, die Staatsschulden niedriger zu halten als beim Zinssatz $r=g$. Man würde dann ein gewisses Ausmaß an dynamischer Ineffizienz in Kauf nehmen. Denn mit den geringeren Staatsschulden geht ein niedrigerer Zinssatz einher.

\section{Intra-generationelle Verteilungseffekte - Fall 2}

Komplizierter wird die Analyse, wenn wir die Boden- und Monopolrenten und ihre Kapitalisierung mit einbeziehen. Da auch dann die Golden Rule gilt, ist es sinnvoll, diese als Referenzpunkt beizubehalten. Ich wiederhole hier die oben schon genannte Formel für die Golden Rule

$$
Z=D+T+\beta l
$$

Diese Formel legt nahe, dass unter Beibehaltung des herrschenden Zinssatzes die kapitalisierten Renten $\beta l$ und die Staatsschulden $D$ eine Art Substitute sind. Denn in erster Approximation mögen wir ja annehmen, dass bei gleichbleibendem Zinssatz sowohl der Vermögenswunsch $Z$ als auch die Kapitalbindung der Produktion $\mathrm{T}$ gleich bleiben. Dann wären die Staatsschulden und die kapitalisierten Renten sogar eine Art perfekter Substitute.

Das erinnert an die These Böhm-Bawerks, der in seiner Habilitationsschrift „Rechte und Verhältnisse in der volkswirtschaftlichen Güterlehre“ (Böhm-Bawerk 1881) die These aufgestellt hat: (modern ausgedrückt): kapitalisierte Monopolrenten (z. B. aus Patentrechten) gehören nicht zum Volksvermögen. Denn auch Forderungen, denen Schulden anderer entsprechen, gehören nicht zum Volksvermögen. Diese These war damals kontrovers. Selbst Böhm-Bawerks Lehrer Carl Menger wollte sich ihr nicht anschließen. Böhm-Bawerks Argument war dieses: den künftigen Monopolrenten entsprechen künftige Preisaufschläge über die Kosten, die sich für die Endverbraucher wirtschaftlich so auswirken wie die Rückzahlung von Schulden. Böhm-Bawerk hat allerdings die kapitalisierten Bodenrenten nicht in sein Verdikt einbezogen. Die Nutzung von Boden führt nach Böhm-Bawerk zu erhöhten Herstellungskosten. Daher seien die Bodenwerte Teil des Volksvermögens.

Diese Rückbesinnung auf Böhm-Bawerk hat ihren guten Sinn deshalb, weil die heutigen Bodenrenten ganz überwiegend städtische Lagerenten sind. Der Verkehrswert des landwirtschaftlichen Bodens ist ein kleiner Teil des Gesamtwerts des Bodens. Nun gibt es seit einigen Jahrzehnten das Arnott-Stiglitz-Theorem (1979) über die städtischen Lagerenten: Im Fall einer Stadt optimaler Bevölkerungsgröße besteht das optimale Volumen und die optimale Finanzierung der lokalen öffentlichen Güter darin, dass die Lagerenten der Stadt zu 100\% steuerlich abgeschöpft werden und damit die Kosten der lokalen öffentlichen Güter zu $100 \%$ abgedeckt werden. In diesem Optimalfall wäre der privatwirtschaftliche Verkehrswert des urbanen Bodens praktisch null. Arnott und Stiglitz nennen ihr Theorem ein „Henry-George-Theo- 
rem“ nach dem amerikanischen Ökonomen Henry George, der ja propagierte, dass aller Boden in Gemeineigentum überführt werden sollte. Ähnliche Thesen vertrat bekanntlich Silvio Gesell mit seiner „Freiland-Theorie“.

In der Wirklichkeit ist der gesellschaftlich optimale private Vermögenswert der Lagerenten vermutlich nicht Null. Denn es erscheint sinnvoll, den „Developer“, der mit seinen Projekten Agrarland oder nicht genutztes Land in volkswirtschaftlich wertvolleres Bauland verwandelt, mit einem Teil des Wertzuwachses zu entlohnen.

Ich definiere nunmehr den Referenzzustand als den, der sowohl bezüglich des Zinssatzes als auch bezüglich der kapitalisierten Lagerenten der optimale ist. Die entsprechenden Größen in unserer Gleichung versehe ich mit einem Stern: *.

$$
Z^{*}=D^{*}+T^{*}+\beta^{*} l^{*}
$$

Nun machen wir folgendes Gedankenexperiment. Wir zerteilen den repräsentativen Haushalt in die Klasse der „Bodenbesitzer“" und die „Nicht-Bodenbesitzer“. Da die beiden Klassen bei den Größen * ihr summiertes Lebenseinkommen maximieren, muss jede Abweichung von diesem Referenzzustand mindestens eine der beiden Klassen schlechter stellen. Wir halten nun den Zinssatz fest. Es bleibt also $r=g$. Jedoch senken wir die Staatsschulden. Wie oben schon erwähnt, können wir davon ausgehen, dass damit auch die Kapitalbindung Tim Produktionsprozess gleich bleibt. Zuerst betrachten wir den Fall, dass bei diesem gleichgebliebenen Zins auch das gewünschte Privatvermögen $Z$ gleich bleibt. In diesem Fall muss mit den gesenkten Staatsschulden auch die kapitalisierte Bodenrente um den gleichen Betrag steigen. Das geht nur, indem $\beta$, also der Rentenanteil an der Wertschöpfung steigt. Die Staatschuldensenkung bedeutete damit eine Verschiebung der Einkommensverteilung zugunsten der Bodenbesitzer und damit zulasten der Nichtbodenbesitzer.

Wir können denselben Tatbestand auch wie folgt ausdrücken: eine mit der Beibehaltung der Golden Rule kompatible steuerliche Entlastung der Bodenbesitzer (relativ zur optimalen Besteuerung der Lagerenten) geht einher mit einer Reduktion der Staatsschulden. Sie begünstigt die Klasse der Bodenbesitzer und belastet die Klasse der Nichtbodenbesitzer.

Es ist realistisch, davon auszugehen, dass der Wohlstand der Besitzer städtischen Bodens im Durchschnitt höher liegt als der Wohlstand der Nichtbodenbesitzer. Damit läuft das eben durchgeführte Gedankenexperiment einer Senkung der Staatsschulden und einer zins-kompensierenden Anhebung der Lagerenten auf eine Erhöhung der Ungleichheit der Wohlstandsverteilung hinaus.

Die Erfahrung lehrt, dass mit dieser Umverteilung von Arm zu Reich auch die Vererbungsquote des Vermögens ansteigt. Immobilien, die im direkten Privatbesitz sind, werden ganz überwiegend an die nachfolgende Generation vererbt - und dies in einer Mehrzahl von Fällen schuldenfrei. (Bei Erbteilungen werden sie dann häufig verkauft, aber eben erst nach dem Erbfall). Eine höhere Vererbungsquote des Vermögens geht aber mit einem höheren durchschnittlichen Vermögenswunsch einher. Die „Warteperiode“ $Z$ steigt somit, wenn bei gleichbleibendem Zinssatz die kapitalisierte Bodenrente steigt. Insofern sinkt die Schuldenquote $D$ um weniger als die kapitalisierte Bodenrente steigt. 
Will man aus Gründen erhöhter „Verteilungsgerechtigkeit“ die Lagerenten sogar stärker besteuern als dem Effizienzmaximum entspricht, dann erfordert dies unter Beibehaltung des optimalen Zinses, dass die Staatsschulden noch höher sind als sie es im Effizienzmaximum wären.

\section{Schlussbemerkung}

Die hier vorgeführten Gedankenexperimente zeigen die Ambivalenz bei den Verteilungswirkungen einer veränderten Staatsverschuldung. Sie legen nahe, dass es unvorsichtig wäre, ohne eine genauere Spezifikation der betrachteten Lage Aussagen über diese Verteilungswirkungen zu machen. Aussagen wie ,Staatsverschuldung ist ungerecht, weil sie künftige Generationen benachteiligt“ oder ,höhere Zinsen verbessern die Lage der unteren Einkommensgruppen“ sind als Pauschalaussagen mit Sicherheit falsch.

Funding Open access funding provided by Max Planck Society.

Open Access Dieser Artikel wird unter der Creative Commons Namensnennung 4.0 International Lizenz (http://creativecommons.org/licenses/by/4.0/deed.de) veröffentlicht, welche die Nutzung, Vervielfältigung, Bearbeitung, Verbreitung und Wiedergabe in jeglichem Medium und Format erlaubt, sofern Sie den/die ursprünglichen Autor(en) und die Quelle ordnungsgemäß nennen, einen Link zur Creative Commons Lizenz beifügen und angeben, ob Änderungen vorgenommen wurden.

\section{Literatur}

Böhm-Bawerk, E. von (1881). Rechte und Verhältnisse vom Standpunkte der volkswirtschaftlichen Güterlehre. Innsbruck.

Böhm-Bawerk, E. von (1889). Kapital und Kapitalzins. Bd. 2. Innsbruck: Abteilung: Positive Theorie des Kapitales.

Diamond, P. (1965). National debt in a neoclassical growth model. American Economic Review, 55, $1126-1150$

Hicks, J.R. (1939). Value and capital: an inquiry into some fundamental principles of economic theory. Oxford: Oxford University Press.

Homburg, S. (1991). Interest and growth in an economy with land. Canadian Journal of Economics, 24, $450-459$.

Phelps, E. (1961). The golden rule of accumulation: a fable for growthmen. American Economic Review, $51,638-643$.

Weizsäcker, C. C. von (1962). Wachstum, Zins und optimale Investitionsquote. Tübingen: Mohr.

Weizsäcker, C.C. von (2015). Langfristiges Wirtschaftswachstum: Was gesagt werden kann und was nicht gesagt werden kann. In H. J. Ramser \& M. Stadler (Hrsg.), Entwicklung und Perspektiven der Wirtschaftswissenschaft (S. 93-100). Tübingen: Mohr Siebeck. 